Check for updates

Cite this: Phys. Chem. Chem. Phys., 2019, 21, 8837

Received 31st January 2019 Accepted 3rd April 2019

DOI: $10.1039 / c 9 c p 00613 c$

rsc.li/pccp

\title{
Destructive role of oxygen in growth of molybdenum disulfide determined by secondary ion mass spectrometry
}

\author{
Paweł Piotr Michałowski, (D)* Piotr Knyps, Paweł Ciepielewski, Piotr Caban, \\ Ewa Dumiszewska and Jacek Baranowski
}

\begin{abstract}
The application of secondary ion mass spectrometry (SIMS) in investigation and comparison of molybdenum disulfide $\left(\mathrm{MOS}_{2}\right)$ films grown on $\mathrm{SiO}_{2}, \mathrm{Al}_{2} \mathrm{O}_{3}$ and $\mathrm{BN}$ substrates is presented. SIMS measurements of the $\mathrm{MoS}_{2}$ /substrate interface reveals oxygen out-diffusion from the substrates containing oxygen and the formation of an amorphous MoOS layer in addition to $\mathrm{MoS}_{2}$. The total area of $\mathrm{MoS}_{2}$ domains covering the substrate is directly related to the type of substrate. For $\mathrm{SiO}_{2}$, small triangular domains of $\mathrm{MOS}_{2}$ separated by amorphous MoOS material are observed. For $\mathrm{Al}_{2} \mathrm{O}_{3}$, the sizes of the $\mathrm{MoS}_{2}$ domains are drastically improved due to the higher stability of sapphire. For a BN substrate, SIMS measurements reveal a uniform $\mathrm{MOS}_{2}$ coverage over the whole 2-inch wafer. These results show the destructive role of oxygen released from substrates such as $\mathrm{SiO}_{2}$ or $\mathrm{Al}_{2} \mathrm{O}_{3}$ during the growth process of $\mathrm{MoS}_{2}$. The fast and cheap growth process on a non-oxide substrate allows large wafer-scale uniform molybdenum disulfide material to be obtained, which is promising for device fabrication.
\end{abstract}

\section{Introduction}

Two-dimensional transition metal dichalcogenides (TMDs), such as $\mathrm{MoS}_{2}, \mathrm{MoSe}_{2}, \mathrm{WS}_{2}, \mathrm{WSe}_{2}$ etc. have received great attention and have emerged as an attractive class of nanomaterials due to their unique structures. ${ }^{1-4}$ Their electronic properties vary with thickness and make them possible candidates for 2D nano-electronic and optoelectronic applications such as solar cells, photodetectors, field effect transistors, and sensors.

Among TMD materials, molybdenum disulfide has been extensively studied. ${ }^{5-13} \mathrm{MoS}_{2}$ is a two-dimensional crystal with strong in-plane covalent bonding and weak out of plane van der Waals interactions. In the monolayer form, $\mathrm{MoS}_{2}$ has a direct energy gap.

The most common way to obtain $\mathrm{MoS}_{2}$ monolayers is by mechanical exfoliation of bulk material. However, this method is not promising for obtaining large wafer-scale uniform areas of $2 \mathrm{D}$ material suitable for device fabrication. There have been several attempts to produce $\mathrm{MoS}_{2}$ layers via chemical vapour deposition (CVD) on insulating substrates. ${ }^{14-16}$ Among CVD methods, the direct growth of 2D materials with the use of thin metal film deposition connected with controlled sulfurization

Institute of Electronic Materials Technology, Wólczyńska 133, 01-919 Warsaw, Poland.E-mail: pawel.michalowski@itme.edu.pl has become one of the promising ways to synthesize large area 2D materials. ${ }^{17,18}$ In the present work, we report successful sulfurization of molybdenum film deposited on different substrates such as $\mathrm{SiO}_{2}, \mathrm{Al}_{2} \mathrm{O}_{3}$ and $\mathrm{BN}$.

Various experimental techniques such as Raman spectroscopy, transmission electron microscopy, scanning probe microscopy, scanning tunnelling microscopy, atomic force microscopy, X-ray diffraction, and X-ray photoelectron spectroscopy have been used to investigate $2 \mathrm{D}$ materials. ${ }^{19-30}$ Each of these methods has its own strengths and limitations, and the information they provide is complementary. In this work, secondary ion mass spectrometry (SIMS) is introduced as a suitable method for characterization of $\mathrm{MoS}_{2}$ layers. The technique is widely used to determine the elemental composition of a sample,$^{31-36}$ however in the case of 2D materials sub-nanometer depth resolution is required. In our previous works we have already established reliable procedures to characterize such samples, namely graphene ${ }^{37-41}$ and boron nitride. $^{42,43}$ These results have inspired us to methodically develop our measurement procedures and adjust them for a wide range of 2D materials, including $\mathrm{MoS}_{2}$.

The main motivation of this work is to investigate the role of substrates such as $\mathrm{SiO}_{2}, \mathrm{Al}_{2} \mathrm{O}_{3}$ and $\mathrm{BN}$ on the quality and size of the grown $\mathrm{MoS}_{2}$ domains. The SIMS measurements play an essential role in this investigation. It is shown that the choice of substrate has a dramatic influence on the size of the grown $\mathrm{MoS}_{2}$ domains. 


\section{Experimental}

\subsection{Sample preparation}

Three kinds of substrate were used for the growth of $\mathrm{MoS}_{2}$ layers:

1. Sample A: $\mathrm{SiO}_{2} / \mathrm{Si}$ (thermal oxide of $200 \mathrm{~nm}$ of $\mathrm{SiO}_{2}$ on a $\mathrm{Si}$ substrate)

2. Sample B: sapphire $\left(\mathrm{Al}_{2} \mathrm{O}_{3}\right)$

3. Sample C: BN grown on $\mathrm{Al}_{2} \mathrm{O}_{3}$

The sizes of all substrates were 2 inches. Boron nitride was grown at $1050{ }^{\circ} \mathrm{C}$ in the self-terminated growth mode, which corresponds to a thickness of $2 \mathrm{~nm}$ by the CVD process described by us previously. ${ }^{42}$ All samples were cleaned in alcohol and DI water. Thin Mo film deposition was done by an e-beam PVD deposition process. Deposition of Mo metal was done under $5 \times 10^{-7}$ Torr pressure with a rate of $0.05 \AA \mathrm{s}^{-1}$. The evaporated Mo film had from 0.4 to $1.0 \mathrm{~nm}$ thickness. The Mo sputtered samples were placed in the high temperature zone of a chemical vapour deposition (CVD) reactor for sulfurization to form $\mathrm{MoS}_{2}$ film. $\mathrm{H}_{2} \mathrm{~S}$ was used as the source of sulfur and $\mathrm{H}_{2}$ as the carrier gas. The furnace was heated up to a temperature of $750{ }^{\circ} \mathrm{C}$ and held for 15 minutes. Afterwards, the growth furnace was naturally cooled to $150{ }^{\circ} \mathrm{C}$ for evaporation of excess sulfur from the surface of the samples. A monolayer of $\mathrm{MoS}_{2}$ exfoliated from a bulk material and transferred on to $\mathrm{SiO}_{2}$ was used as a reference sample.

\subsection{SIMS measurements}

In this work, all SIMS measurements were performed employing a CAMECA SC Ultra instrument under ultra-high vacuum (UHV), usually of $4 \times 10^{-10}$ mbar. The $\mathrm{Cs}^{+}$primary beam was rastered over $80 \times 80 \mu \mathrm{m}^{2}$ (the analysis area was limited to $50 \times 50 \mu \mathrm{m}^{2}$ ) and positive ions detection mode was used in the experiments and thus all species were measured as $\mathrm{CsX}^{+}$cluster ions. The intensity of the primary beam was $4 \mathrm{nA}$ and the impact energy was $150 \mathrm{eV}$. For precise oxygen detection measurements, secondary ions detection mode was switched to negative but the rest of the parameters remained the same. To avoid charging problems the electron gun was used. A highly uniform beam was required for this work - the beam on the sample in the SC Ultra tool has a square shape and owning to the "variable rectangular shape concept" forms a homogeneous spot. The primary beam at the working point in the SC Ultra is formed by two stencils - wellshaped apertures. While the first one is used to choose the most intense and homogeneous part of the Gaussian-shaped ion beam, the second one changes the size of the spot. This innovation provides high sensitivity for all measured elements. ${ }^{44-46}$

The lateral imaging mode was very important in these experiments. This task was, however, very challenging. The intensity of SIMS signals in the $\mathrm{CsX}^{+}$mode depended predominantly on cesium deposition at the surface of the sample. In the depth profiling mode, ions were collected from a large area and thus the average intensity was very stable. For the imaging mode each point was measured individually and thus some significant fluctuations might decrease the quality of measurements. Indeed, we performed standard imaging measurements
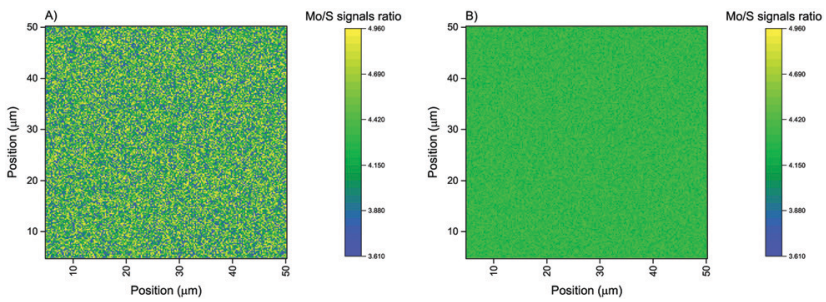

Fig. 1 SIMS distribution maps for the reference sample. (A) The standard imaging procedure showed significant fluctuations; (B) the refined procedure increases the quality of measurements and is suitable for identification of the $\mathrm{MoS}_{2}$ phase.

on the reference sample - see Fig. $1 \mathrm{~A}$ - and found that while the average ratio of the Mo and S signals was 4.313 (just like it was in the depth profiling mode), the fluctuations were as high as $30 \%$. It was concluded that a proper identification of the $\mathrm{MoS}_{2}$ phase would be impossible for this kind of experiment.

To solve this issue, the source of these fluctuations was identified. The primary beam was very uniform and thus a lateral distribution of cesium at the surface of a sample should have inherited this characteristic. The problem was that the magnetic sector SIMS can only measure one ion at a time. In the depth profiling mode, it was not an important issue as the integration time for each signal was about one second, so each cycle was as follows: integration of the S signal, adjusting the electromagnet for different ion mass (during this phase the primary beam was blanked and the sample was not sputtered), integration of the Mo signal and once again adjusting the electromagnet. It meant that there was only one second of sputtering time difference between the integration of the $\mathrm{S}$ and Mo signals, and thus it was not expected that the cesium deposition changed significantly within that time. Such a short integration time could be used because ions were collected from a relatively large area. Lateral imaging, however, required much longer integration time to achieve similar sensitivity - in this particular case each signal was integrated for fifteen seconds to form a distribution map. During that time the cesium deposition might have changed a lot and thus the $\mathrm{Mo} / \mathrm{S}$ ratio fluctuated a lot. To solve this problem we changed the measurement procedure: each signal was sequentially integrated for about 0.3 seconds and fifty cycles were summed to form a distribution map so that the total integration time of each signal was fifteen seconds. Such a procedure is significantly more time consuming as it introduced fifty times more electromagnet adjusting phases (each of them lasted for about two seconds) during which the sample was not sputtered, but it increased the quality of measurements. Indeed, measurements on the reference sample with this refined procedure - see Fig. 1B - showed that the fluctuations were reduced to about $6 \%$ which was acceptable for identification of molybdenum disulfide. Based on these results, we assumed that the ratio $\mathrm{Mo} / \mathrm{S}=4.313 \pm 3 \%$ was a marker of the $\mathrm{MoS}_{2}$ phase.

\subsection{Raman spectroscopy measurements}

Room temperature Raman measurements were performed with a Renishaw inVia Raman microscope using a $532 \mathrm{~nm}$ wavelength 
obtained from a Nd:YAG laser. The size of a laser spot was about $0.5 \mu \mathrm{m}$ and the power was below $0.1 \mathrm{~mW}$ to avoid the destruction of $\mathrm{MoS}_{2}$ layers. The laser was focused on the sample using a $\times 100$ objective and numerical aperture NA $=0.9$ in a backscattering geometry.

\section{Results \& discussion}

When a new growth procedure is being established and optimized it is usually advisable to test it on a cheap, easily accessible substrate. The growth of $\mathrm{MoS}_{2}$ films on $\mathrm{SiO}_{2}$ substrates meets this criterion and provides the additional advantage of possible direct integration of a novel 2D material with the existing silicon-based fabrication lines. However, the influence of the substrate on the growth process is often omitted. The SIMS imaging mode shows that the quality of the obtained film is poor - Fig. 2A and B presents lateral distributions of the $\mathrm{Mo} / \mathrm{S}$ ratio close to the surface and the substrate, respectively. As was determined on a reference sample, the ratio of $\mathrm{Mo} / \mathrm{S}$ signals equals 4.313 for $\mathrm{MoS}_{2}$ material. It can be immediately noted that the proposed measurement procedure is effective as triangles of molybdenum disulfide can be identified, and this material is particularly known for formation of such triangular domains. ${ }^{47-50}$ Experiments performed on several different spots confirmed that for layers closer to the surface the size of the $\mathrm{MoS}_{2}$ domains is bigger.

Outside of these triangular domains, the ratio of $\mathrm{Mo} / \mathrm{S}$ signals is chaotic but the average value is below 4.313. Some points are close to this value, but it should be noted that the lateral resolution of the experiment is about one micron and thus these points should not be treated as very small domains of $\mathrm{MoS}_{2}$. The only valid conclusion is that these regions contain more sulfur than molybdenum disulfide. To gather more information we have checked whether some other elements are present in this region. Our analysis - see Fig. 2C and $\mathrm{D}$ - reveals that these regions are oxidized. It should be noted
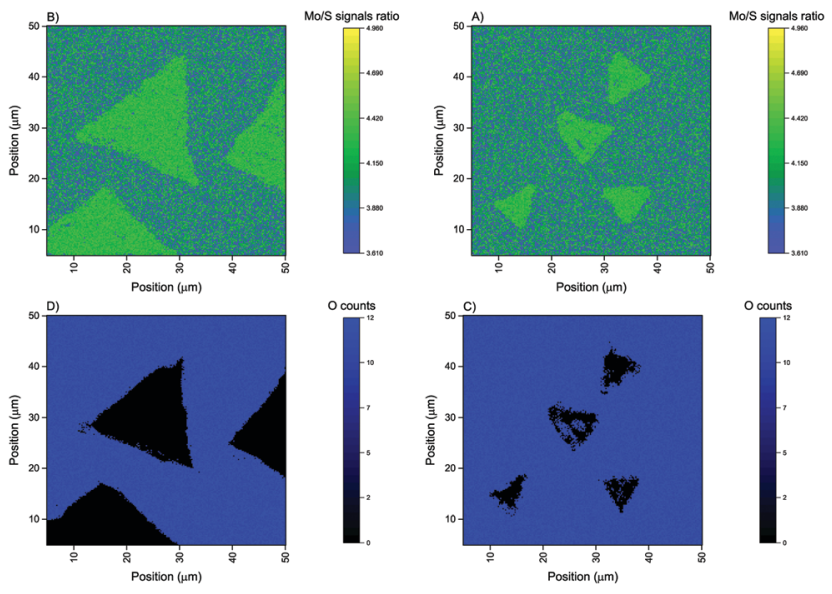

Fig. 2 SIMS distribution maps for sample A. Maps (A) and (B) show the $\mathrm{Mo} / \mathrm{S}$ distribution close to the surface and the substrate, respectively (counting from the surface) while (C) and (D) show oxygen distribution for the same layers. Bigger domains are formed close to the surface. that the $\mathrm{CsX}^{+}$mode is not sensitive for oxygen contamination when some oxygen counts are registered it can be concluded that these regions contain at least several atomic percent of this element. The lateral resolution of this element is worse than in the case of Mo and S, as oxygen ions are lighter and thus have a broader energy distribution and it is more difficult to effectively filter ions coming from neighboring regions. Nevertheless, the quality is good enough to show that no/little oxygen is found in the triangular $\mathrm{MoS}_{2}$ domains, which is particularly well visible for bigger triangles.

At this point, we were not able to determine what the source of oxygen that has oxidized the sample was. In theory, the film could have oxidized during the time it was transferred from the reactor to the SIMS tool. Thus, we decided to compare the quality of $\mathrm{MoS}_{2}$ film grown under the same growth conditions but on a different substrate. For that, we chose another easily accessible substrate, namely sapphire, and found that the quality of $\mathrm{MoS}_{2}$ films significantly increased. Fig. 3A shows the distribution of the $\mathrm{Mo} / \mathrm{S}$ ratio for sample $\mathrm{B}$. There is no difference between the region close to the surface and to the substrate - they look exactly the same. Even though the film is not perfectly uniform, the domains of $\mathrm{MoS}_{2}$ cover more than $90 \%$ of the sample. Boundaries between these domains contain more sulfur and are oxidized - see Fig. 3B. However, it should be noted that the width of these boundaries is artificially stretched, since when the primary beam is located partially on a $\mathrm{MoS}_{2}$ region and partially on the boundary the registered $\mathrm{Mo} / \mathrm{S}$ ratio will be lower, and the whole region will be marked as molybdenum sulfide with unknown stoichiometry. Given that the average width of these boundaries is about 2-2.5 micron and the size of a primary beam is about 0.9 micron, it can be concluded that the actual width of the boundaries is in the range of 200-700 $\mathrm{nm}$.

Another possibility is that residual water present at the surface of the samples is responsible for oxidation of the molybdenum sulfide film. We have prepared several samples grown on both types of substrate but with varying vacuum annealing conditions (temperature, time) but no differences have been found. It is important to emphasize that the only difference between samples A and B is the type of substrate. The growth conditions and transfer time from the reactor to the SIMS tool have been as similar as possible. Therefore, this result reveals that the substrate itself has a crucial impact on the quality of the molybdenum sulfide film. It is not surprising
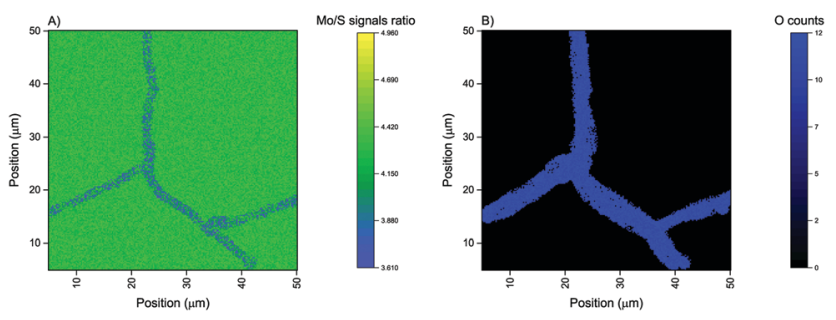

Fig. 3 SIMS distribution maps for sample B. Map (A) shows Mo/S and (B) shows oxygen distribution. 


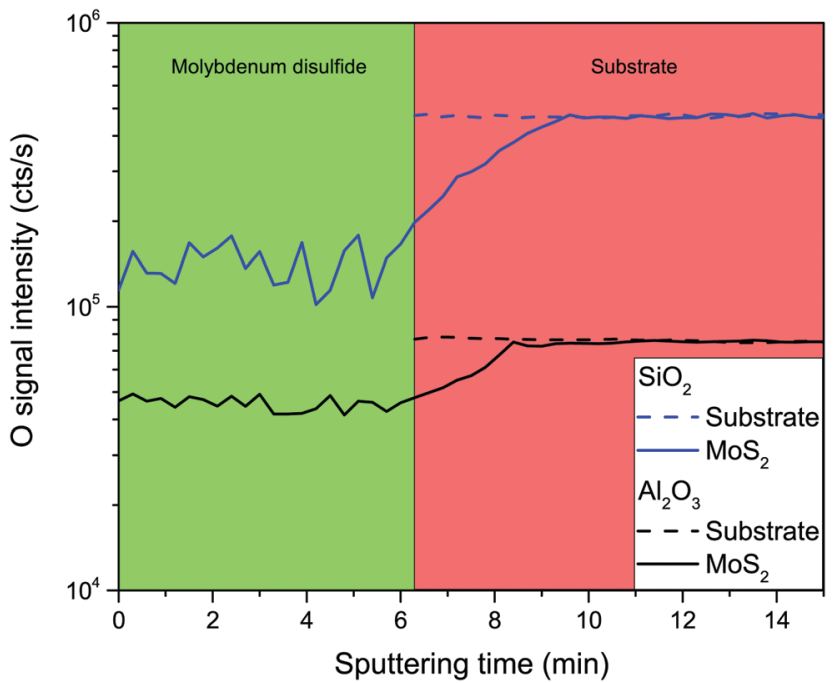

Fig. 4 Oxygen profiles for $\mathrm{MoS}_{2}$ films grown on $\mathrm{SiO}_{2}$ and $\mathrm{Al}_{2} \mathrm{O}_{3}$. The oxygen profiles are similar for both substrates: a clear oxygen depletion from the interface region inside of the substrates is observed. The location of the interface between $\mathrm{MoS}_{2}$ and the substrates has been identified at the point where Si or Al signals increase rapidly (these SIMS signals are not presented in the figure to achieve a better visibility).

that the quality of $\mathrm{MoS}_{2}$ film is much better for sapphire, as this oxide is more stable. The only remaining question is at which point of the growth procedure is oxygen from the substrate incorporated into the molybdenum sulfide film. To answer this question, we have performed additional depth profiling experiments in the negative mode which is more suitable for oxygen detection. For each type of substrate we have compared three different samples: pure substrate, Mo film before the sulfurization process and molybdenum sulfide film. Mo film is not interesting - there is some residual oxygen (in the range of $\mathrm{ppm}$ ) but no impact of the substrate can be detected. Molybdenum sulfide film is by far more interesting. Fig. 4 presents the results of these experiments - as it has been confirmed before, a lot of oxygen can be detected in these samples but the most important finding is the interface region: it can be clearly seen that, after sputtering the whole molybdenum sulfide film, the oxygen signal is still below the level which is typical for the substrate, which means that several nanometers of the substrate is oxygen depleted. It can be therefore concluded that during the sulfurization process at $750{ }^{\circ} \mathrm{C}$ some oxygen is released from the substrate and incorporated into the film, decreasing the overall quality of the sample. We have performed additional experiments where Mo film is heated in a CVD reactor but without introduction of $\mathrm{H}_{2} \mathrm{~S}$, and oxygen depletion in the substrate has been also found (similarly to what is presented in Fig. 4). This means that at high temperature Mo atoms have a tendency to absorb oxygen from a substrate.

It was therefore deemed beneficial to repeat these experiments for another substrate which does not contain any oxygen, namely boron nitride. Fig. 5 presents a lateral imaging of the Mo/S ratio for a molybdenum sulfide film grown on a $\mathrm{BN} / \mathrm{Al}_{2} \mathrm{O}_{3}$ substrate

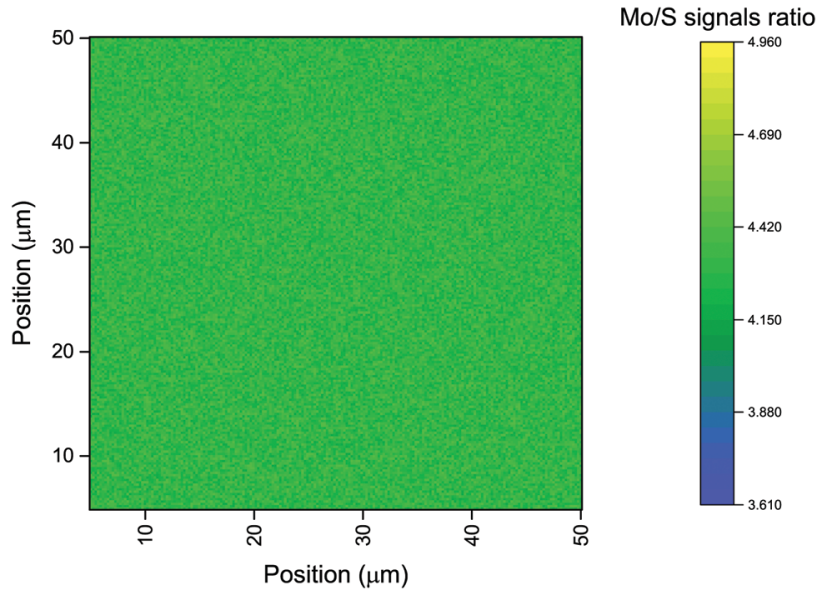

Fig. 5 SIMS distribution map for sample C. The map shows the Mo/S distribution. The sample is very uniform and can be identified as stoichiometric $\mathrm{MoS}_{2}$.

(sample C). It becomes immediately apparent that the film is very uniform and the quality is comparable to the reference sample (see Fig. 1B). Furthermore, no oxygen can be detected in this sample. We have repeated these measurements on 40 spots over the whole 2-inch sample. In all these areas the uniform $\mathrm{MoS}_{2}$ film has been detected without any exception. Therefore it can be concluded that the whole area of the 2 -inch $\mathrm{BN} / \mathrm{Al}_{2} \mathrm{O}_{3}$ substrate is covered by $\mathrm{MoS}_{2}$ film.

Finally, we confirmed the quality of these samples with Raman spectroscopy, a powerful nondestructive characterization tool which is widely used to characterize 2D materials. Specifically, in the case of ultrathin $\mathrm{MoS}_{2}$ it is used to identify the number of layers, as it has been demonstrated by $\mathrm{Li}$ et $a .^{51}$ that the frequencies of the Raman $\mathrm{E}_{2 \mathrm{~g}}^{1}$ and $\mathrm{A}_{1 \mathrm{~g}}$ peaks are strongly dependent on the $\mathrm{MoS}_{2}$ thickness in the range of 1-4 monolayers. The frequency difference of these modes increases from about $19 \mathrm{~cm}^{-1}$ for a monolayer to $25 \mathrm{~cm}^{-1}$ for a bulk material.

Fig. 6 compares the Raman spectra of samples A, B and C. It can be immediately noted that the quality of the sample $\mathrm{A}$ is poor - both peaks are broad and of very low intensity (the Raman signal was collected 5 times longer with the same laser power). The frequency difference is about $24 \mathrm{~cm}^{-1}$ which indicates four layers of $\mathrm{MoS}_{2}$. Sample B is of much better quality.
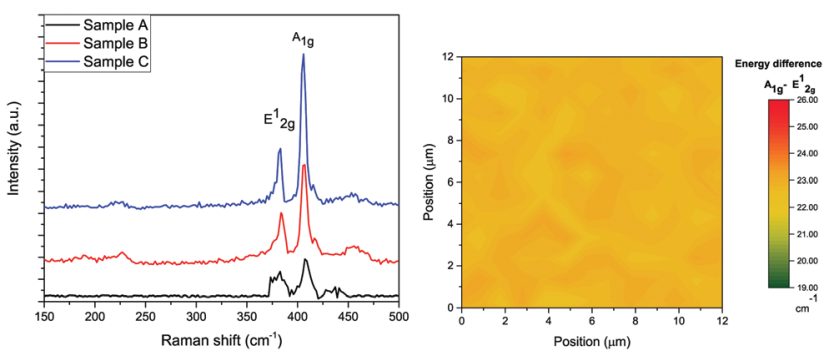

Fig. 6 Raman spectra of samples A, B and C. Changing the substrate from silicon dioxide to sapphire significantly increased the quality of the $\mathrm{MoS}_{2}$, but the sample grown on boron nitride was the best one. The Raman map shows the energy difference between the $E_{2 g}^{1}$ and $A_{1 g}$ peaks for sample $C$. 
Both peaks are sharp and intense. The frequency difference is about $22 \mathrm{~cm}^{-1}$ which indicates two layers of $\mathrm{MoS}_{2}$. Sample C is confirmed to be of the highest quality, and the frequency difference is about $23 \mathrm{~cm}^{-1}$ which indicates three layers. The map of the frequency difference between $E_{2 g}^{1}$ and $A_{1 g}$ peaks indicates that the average thickness of the $\mathrm{MoS}_{2}$ film is uniform indeed.

\section{Conclusions}

The most important result of our investigation is a comparison of the $\mathrm{MoS}_{2}$ coverage area on different types of substrates. It has been found that the coverage is dramatically influenced by the choice of type of substrate, such as $\mathrm{SiO}_{2}, \mathrm{Al}_{2} \mathrm{O}_{3}$, or BN. SIMS measurements have revealed the release of oxygen from a substrate such as $\mathrm{SiO}_{2}$ or $\mathrm{Al}_{2} \mathrm{O}_{3}$, which has a drastic influence on the size of the grown $\mathrm{MoS}_{2}$ domains. During the sulfurization of Mo, the oxygen out-diffuses from a substrate and reacts with Mo, leading to formation of an amorphous MoOS material of unknown stoichiometry. In the case of growth on a $\mathrm{SiO}_{2}$ substrate, a relatively large amount of oxygen was released, leading to the formation of a large area of amorphous MoOS covering about $50 \%$ of the total area. The presence of MoOS prevented enlargement of the $\mathrm{MoS}_{2}$ triangle domains. On the other hand, sapphire is a much more stable oxide then $\mathrm{SiO}_{2}$ and, therefore, a smaller amount of oxygen may go into the grown layer, which led to much larger $\mathrm{MoS}_{2}$ domains separated by narrow oxidized boundaries. In this case, about $90 \%$ of the whole area was covered by $\mathrm{MoS}_{2}$ domains and only $10 \%$ by oxygen-rich amorphous MoOS. Some substrates such as $\mathrm{BN}$ do not contain oxygen at all. The $\mathrm{BN}$ layer grown on $\mathrm{Al}_{2} \mathrm{O}_{3}$ separates the sapphire, and apparently blocks oxygen diffusion to the grown $\mathrm{MoS}_{2}$ film. In this case, $100 \%$ of the $\mathrm{BN} 2$-inch wafer was covered by continuous and uniform $\mathrm{MoS}_{2}$.

Let us emphasize the key role of SIMS measurements in our investigation. SIMS determination of the presence of oxygen at the $\mathrm{MoS}_{2}$ /substrate interface has disclosed the process of oxygen out-diffusing from oxide substrates. This process is destructive from the perspective of growth of $\mathrm{MoS}_{2}$ domains, due to the formation of MoOS material which prohibits the formation of a large and continuous $\mathrm{MoS}_{2}$ film. The BN substrate, being free from oxygen, is the optimal one for obtaining continuous wafer-scale $\mathrm{MoS}_{2}$ film.

Generalizing, the presented SIMS results show the invalidity of the assumption that a substrate does not react with a grown layer in CVD growth. Such a reaction can go beyond simple contamination and can determine the size of the grown $\mathrm{MoS}_{2}$ domains. The choice of an oxide-free substrate for the growth of $\mathrm{MoS}_{2}$, and most likely for other TMDs, is of fundamental importance especially when device fabrication is considered.

\section{Conflicts of interest}

There are no conflicts to declare.

\section{Acknowledgements}

This work was supported by the European Union's Horizon 2020 research and innovation programme under grant agreement no. 785219.

\section{References}

1 W. S. Yun, S. W. Han, S. C. Hong, I. G. Kim and J. D. Lee, Phys. Rev. B: Condens. Matter Mater. Phys., 2012, 85, 033305.

2 D. W. Bullett, J. Phys. C: Solid State Phys., 1978, 11, 4501.

3 Q. H. Wang, K. Kalantar-Zadeh, A. Kis, J. N. Coleman and M. S. Strano, Nat. Nanotechnol., 2012, 7, 669.

4 X. Song, J. Hu and H. Zeng, J. Mater. Chem. C, 2013, 1, 2952.

5 S. Ding, D. Zhang, J. S. Chen and X. W. D. Lou, Nanoscale, 2012, 4, 95.

6 Y. Li, H. Wang, L. Xie, Y. Liang, G. Hong and H. Dai, J. Am. Chem. Soc., 2011, 133, 7296.

7 G. L. Yu, R. Jalil, B. Belle, A. S. Mayorov, P. Blake, F. Schedin, S. V. Morozov, L. A. Ponomarenko, F. Chiappini, S. Wiedmann, U. Zeitler, M. I. Katsnelson, A. K. Geim, K. S. Novoselov and D. C. Elias, Proc. Natl. Acad. Sci. U. S. A., 2013, 110, 3282.

8 S. Wi, H. Kim, M. Chen, H. Nam, L. J. Guo, E. Meyhofer and X. Liang, ACS Nano, 2014, 8, 5270.

9 A. K. Geim and I. V. Grigorieva, Nature, 2013, 499, 419.

10 M. Chhowalla, H. Shin, G. Eda, L.-J. Li, K. Loh and H. Zhang, Nat. Chem., 2013, 5, 263.

11 W. Wu, L. Wang, Y. Li, F. Zhang, L. Lin, S. Niu, D. Chenet, X. Zhang, Y. Hao, T. Heinz, J. Hone and Z. Wang, Nature, 2014, 514, 470.

12 R. Ganatra and Q. Zhang, ACS Nano, 2014, 8, 4074.

$13 \mathrm{X}$. Li and H. Zhu, J. Materiomics, 2015, 1, 33.

14 X. Wang, H. Feng, Y. Wu and L. Jiao, J. Am. Chem. Soc., 2013, 135, 5304.

15 Y.-H. Lee, X.-Q. Zhang, W. Zhang, M.-T. Chang, C.-T. Lin, K.-D. Chang, Y.-C. Yu, J. T.-W. Wang, C.-S. Chang, L.-J. Li and T.-W. Lin, Adv. Mater., 2012, 24, 2320.

16 Y. Zhan, Z. Liu, S. Najmaei, P. M. Ajayan and J. Lou, Small, 2012, 8, 966.

17 M. H. Heyne, D. Chiappe, J. Meersschaut, T. Nuytten, T. Conard, H. Bender, C. Huyghebaert, I. P. Radu, M. Caymax, J.-F. de Marneffe, E. C. Neyts and S. De Gendt, J. Mater. Chem. C, 2016, 4, 1295.

18 A. Stesmans, S. Iacovo, D. Chiappe, I. Radu, C. Huyghebaert, S. De Gendt and V. V. Afanas'ev, Nanoscale Res. Lett., 2017, 12, 283.

19 L. Malard, M. Pimenta, G. Dresselhaus and M. Dresselhaus, Phys. Rep., 2009, 473, 51.

20 G. Plechinger, S. Heydrich, J. Eroms, D. Weiss, C. Schüller and T. Korn, Appl. Phys. Lett., 2012, 101, 101906.

21 A. Ferrari and D. Basko, Nat. Nanotechnol., 2013, 8, 235.

22 X. Ming, Int. J. Spectrosc., 2018, 2018, 4861472.

23 M. Paillet, R. Parret, J.-L. Sauvajol and P. Colomban, J. Raman Spectrosc., 2018, 49, 8.

24 S. Zhang, N. Zhang, Y. Zhao, T. Cheng, X. Li, R. Feng, H. Xu, Z. Liu, J. Zhang and L. Tong, Chem. Soc. Rev., 2018, 47, 3217. 
25 F. Liang, H. Xu, X. Wu, C. Wang, C. Luo and J. Zhang, Chin. Phys. B, 2018, 27, 037802.

26 C. N. R. Rao and A. Nag, Eur. J. Inorg. Chem., 2015, 4244.

27 S. Z. Butler, S. M. Hollen, L. Cao, Y. Cui, J. A. Gupta, H. R. Gutiérrez, T. F. Heinz, S. S. Hong, J. Huang, A. F. Ismach, E. Johnston-Halperin, M. Kuno, V. V. Plashnitsa, R. D. Robinson, R. S. Ruoff, S. Salahuddin, J. Shan, L. Shi, M. G. Spencer, M. Terrones, W. Windl and J. E. Goldberger, ACS Nano, 2013, 7, 2898.

28 C. N. R. Rao, H. S. S. Ramakrishna Matte and U. Maitra, Angew. Chem., Int. Ed., 2013, 52, 13162.

29 G. R. Bhimanapati, Z. Lin, V. Meunier, Y. Jung, J. Cha, S. Das, D. Xiao, Y. Son, M. S. Strano, V. R. Cooper, L. Liang, S. G. Louie, E. Ringe, W. Zhou, S. S. Kim, R. R. Naik, B. G. Sumpter, H. Terrones, F. Xia, Y. Wang, J. Zhu, D. Akinwande, N. Alem, J. A. Schuller, R. E. Schaak, M. Terrones and J. A. Robinson, ACS Nano, 2015, 9, 11509. 30 C. Rao and U. Maitra, Annu. Rev. Mater. Res., 2015, 45, 29. 31 C. A. Andersen and J. R. Hinthorne, Science, 1972, 175, 853. 32 A. Benninghoven, Surf. Sci., 1975, 53, 596.

33 A. Benninghoven, F. G. Rudenauer and H. W. Werner, Secondary ion mass spectrometry: basic concepts, instrumental aspects, applications and trends, John Wiley \& Sons, New York, 1987.

34 H. W. Werner, Surf. Sci., 1975, 47, 301.

35 H. Liebl, J. Vac. Sci. Technol., A, 1975, 12, 385.

36 H. Liebl, J. Appl. Phys., 1967, 38, 5277.

37 P. P. Michałowski, W. Kaszub, A. Merkulov and W. Strupinski, Appl. Phys. Lett., 2016, 109, 011904.

38 P. P. Michałowski, W. Kaszub, I. Pasternak and W. Strupinski, Sci. Rep., 2017, 7, 7479.
39 P. P. Michałowski, I. Pasternak and W. Strupinski, Nanotechnology, 2018, 29, 015702.

40 P. P. Michałowski, I. Pasternak, P. Ciepielewski, F. Guinea and W. Strupinski, Nanotechnology, 2018, 29, 305302.

41 J. Grzonka, I. Pasternak, P. P. Michałowski, V. Kolkovsky and W. Strupinski, Appl. Surf. Sci., 2018, 447, 582.

42 P. A. Caban, D. Teklinska, P. P. Michalowski, J. Gaca, M. Wojcik, J. Grzonka, P. Ciepielewski, M. Mozdzonek and J. M. Baranowski, J. Cryst. Growth, 2018, 498, 71.

43 P. P. Michałowski, P. Caban and J. Baranowski, J. Anal. At. Spectrom., 2019, DOI: 10.1039/C9JA00004F.

44 CAMECA, Genneviliers, CAMECA SC-Ultra, User's Guide, 2005.

45 D. Kouzminov, A. Merkulov, E. Arevalo and H. J. Grossmann, Surf. Interface Anal., 2013, 45, 345.

46 A. Merkulov, Surf. Interface Anal., 2013, 45, 90.

47 R. Ionescu, W. Wang, Y. Chai, Z. Mutlu, I. Ruiz, Z. Favors, D. Wickramaratne, M. Neupane, L. Zavala, R. Lake, M. Ozkan and C. S. Ozkan, IEEE Trans. Nanotechnol., 2014, 13, 749.

48 Z. Lin, M. T. Thee, A. L. Elías, S. Feng, C. Zhou, K. Fujisawa, N. Perea-López, V. Carozo, H. Terrones and M. Terrones, APL Mater., 2014, 2, 092514.

49 W. Chen, J. Zhao, J. Zhang, L. Gu, Z. Yang, X. Li, H. Yu, X. Zhu, R. Yang, D. Shi, X. Lin, J. Guo, X. Bai and G. Zhang, J. Am. Chem. Soc., 2015, 137, 15632.

50 Z. Cheng, M. Xia, R. Hu, C. Liang, G. Liang and S. Zhang, J. Cryst. Growth, 2017, 480, 6.

51 H. Li, Q. Zhang, C. C. R. Yap, B. K. Tay, T. H. T. Edwin, A. Olivier and D. Baillargeat, Adv. Funct. Mater., 2012, 22, 1385. 